\title{
A Novel Modeling Method for Cooperative Multi-robot Systems Using Fuzzy Timed Agent Based Petri Nets*
}

\author{
Hua $\mathrm{Xu}^{1}$ and Peifa $\mathrm{Jia}^{1,2}$ \\ ${ }^{1}$ State Key Lab of Intelligent Technology and Systems, \\ Tsinghua University, Beijing, 100084, P.R. China \\ ${ }^{2}$ Department of Computer Science and Technology, \\ Tsinghua University, Beijing, 100084, P.R. China \\ \{xuhua, dcsjpf\}@mail.tsinghua.edu.cn
}

\begin{abstract}
This paper proposes a cooperative multi-robot system (CMRS) modeling method called fuzzy timed agent based Petri nets (FTAPN), which has been extended from fuzzy timed object-oriented Petri net (FTOPN). The proposed FTAPN can be used to model and illustrate both the structural and dynamic aspects of CMRS. Supervised learning is supported in FTAPN. As a special type of high-level object, agent is introduced, which is used as a common modeling object in FTAPN models. The proposed FTAPN can not only be used to model CMRS and represent system aging effect, but also be refined into the object-oriented implementation easily. At the same time, it can also be regarded as a conceptual and practical artificial intelligence (AI) tool for multi-agent system (MAS) into the mainstream practice of software development.
\end{abstract}

Keywords: Fuzzy, Agent, Petri nets, Object-oriented, Multi-robot system.

\section{Introduction}

Characterized as cooperation and high efficiency, cooperative multi-robot systems (CMRS) have emerged as usual manufacturing equipments in current industries [1]. Differing from generic control systems, the cooperation needs to be considered in the realization of CMRS [1]. So the system modeling, analysis and refinement always meet with difficulties. CMRS can be regarded as a typical multi-agent system (MAS) in distributed artificial intelligence [2]. For modeling MAS, object-oriented methodology has been tried and some typical agent objects have been proposed, such as active object, etc [3]. However, agent based object models still can not depict its structure and dynamic aspects, such as cooperation, learning, temporal constraints, etc [2].

This paper proposes a high level PN called fuzzy timed agent based Petri net (FTAPN) on the base of FTOPN [4] and it is organized as the following. Section 2 reviews the concept of FTOPN and extends FTOPN to FTAPN on the base of

\footnotetext{
* This work is jointly supported by the National Nature Science Foundation (Grant No: 60405011, 60575057) and the China Postdoctoral Foundation for China Postdoctoral Science Fund (Grant No: 20040350078).
} 
ACTALK model. Section 4 uses FTAPN to model a typical CMRS in the wafer etching procedure of circuit industry and makes some modeling analysis to demonstrate its benefits in modeling MAS. Finally, the conclusion and future work can be found in section 5 .

\section{Basic Concepts}

\subsection{FTOPN}

Definition 1. FTOPN is a six-tuple, FTOPN= (OIP, ION, DD, SI, R, I) where OIP, ION, DD and SI are the same as those in HOONet [5] and TOPN [6] and

1) $\mathrm{R}:\{\mathrm{OIP}\} \rightarrow \mathrm{r}$, where $\mathrm{r}$ is a specific threshold.

2) $\mathrm{I}$ is a function of the time $v$. It evaluates the resulting degree of the abstract object firing.

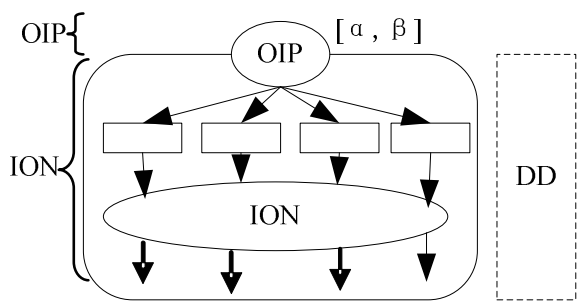

Fig. 1. The General Structure of FTOPN

\subsection{Agent Object and FTAPN}

The active object concept [3] has been proposed to describe a set of entities that cooperate and communicate through message passing. ACTALK is a framework for implementing and computing various active object models into one object-oriented language realization. In ACTALK, an active object is composed of three component classes: address, activity and activeObject [3].

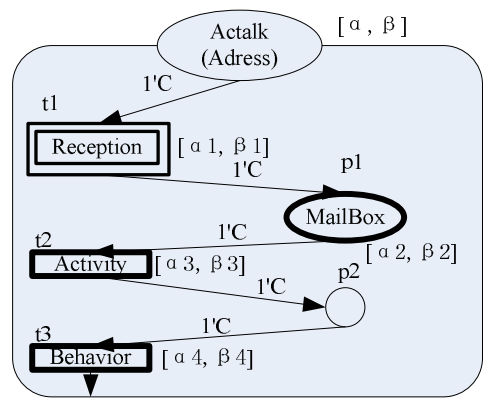

Fig. 2. The FTOPN Model of ACTALK 
The parameters of FTAPN are always given beforehand. In general, however, these parameters may not be available and need to be estimated just like those in FTPN [7]. The estimation is conducted on the base of some experimental data concerning marking of input and output places. The marking of the places is provided as a discrete time series. More specifically we consider that the marking of the output place(s) is treated as a collection of target values to be followed during the training process. As a matter of fact, the learning is carried out in a supervised mode returning to these target data. The learning method is just like those in FTOPN [4].

\section{A Modeling Example}

\subsection{A CMRS Model}

In the etching tools, usually there is a CMRS platform made up of two transferring robots. These two cooperative robots are up to complete transferring one unprocessed wafer from the input lock to the chamber and fetch the processed wafer to the output lock. Any robot can be used to complete the transferring task at any time. If one robot is up to transfer one new wafer, the other will conduct the other fetching task. They will not conflict with each other. Fig. 3 depicts this CMRS FTAPN model, where two agent objects (ACTALK) is used to represent these two cooperative robots. Fig.4 depicts the time relevance rules.

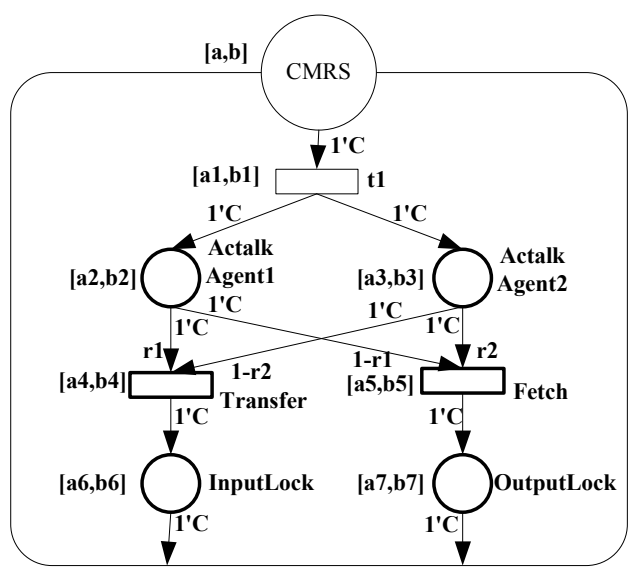

(a) The Agent Based FTAPN Model

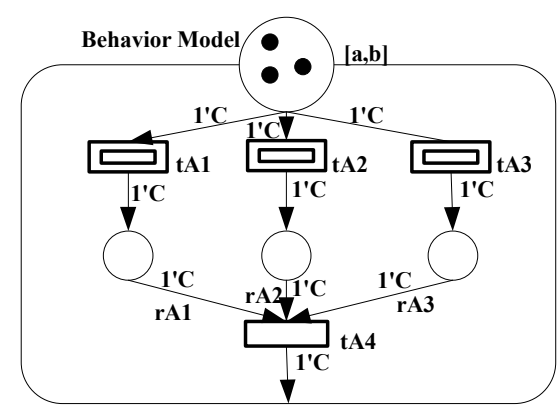

(b) The Behavior Model in Every Agent

Fig. 3. The FTAPN Model

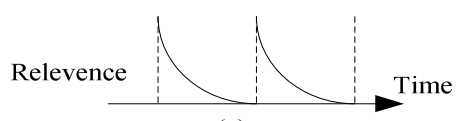

(a)

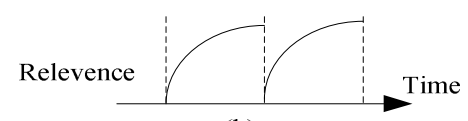

(b)

Fig. 4. The Relevance 


\section{Conclusions}

Cooperative multi robot system is a kind of CMRS in manufacturing industries. In order to model, analyze and simulate this kind of CMRS, this paper proposes fuzzy timed agent based Petri net (FTAPN) on the base of FTOPN [4] and FTPN [7]. In FTAPN, one of the active objects - ACTALK is introduced and used as the basic agent object to model CMRS. Every abstract object in FTOPN can be trained and reduced independently according to the modeling and analysis requirements for $\mathrm{OO}$ concepts supported in FTOPN. The validity of this modeling method has been used to model the CMRS platform in etching tools. The FTAPN can not only model complex MAS, but also be refined into the object-oriented implementation easily. It has provided a methodology to overcome the development problems in agent-oriented software engineering. At the same time, it can also be regarded as a conceptual and practical artificial intelligence (AI) tool for integrating MAS into the mainstream practice of software development.

\section{References}

[1] Cao, Y.U., Fukunaga, A.S., Kahng, A.B., Meng, F.: Cooperative Mobile Robotics: Antecedents and Directions, Autonomous Robots, 4(1997)7-27

[2] Jennings, N.R., Sycara, K., Wooldridge, M.: A Roadmap of Agent Research and Development. Autonomous Agents and Multi-Agent Systems, 1 (1998)7-38

[3] Guessoum, Z., Briot, J.-P., From active objects to autonomous agents, IEEE Concurrency, 7-3(1999) $68-76$

[4] Hua, X., Peifa, J., Fuzzy Timed Object-Oriented Petri Net; Artificial Intelligence Applications and Innovations II-Proceedings of AIAI2005, Berlin Heidelberg New York (2005) 155-166

[5] Hong, J.E., Bae, D.H.: Software Modeling and Analysis Using a Hierarchical Objectoriented Petri net. Information Sciences, 130(2000)133-164

[6] Xu, H.; Jia, P.F.: Timed Hierarchical Object-Oriented Petri Net-Part I: Basic Concepts and Reachability Analysis, Proceedings of RSKT2006, Vol.4062, Lecture Notes In Artificial Intelligence, Berlin Heidelberg New York (2006)727-734

[7] Pedrycz, W.: Camargo, H.: Fuzzy timed Petri nets, Fuzzy Sets and Systems,140(2003): 301-330 